\title{
Density matrix approach to the complex heavy ion optical potential: Exchange part
}

\author{
R. Sartor and Fl. Stancu \\ Institut de Physique, Université de Liège, B-4000 Liege 1, Belgium
}

(Received 25 October 1983)

\begin{abstract}
We further analyze the validity of the density matrix expansion applied to the complex heavy ion optical potential by separately treating the direct and exchange contributions. We show that the discrepancies found previously when comparing the density matrix expansion optical potential with the exact one are mainly to be ascribed to the direct contribution. For the exchange part the density matrix expansion is nearly exact in the tail region and remains very good down to zero separation distance. The Campi-Bouyssy prescription for accelerating the density matrix expansion convergence is also examined.
\end{abstract}

\section{INTRODUCTION}

The microscopic nuclear matter approach ${ }^{1-4}$ to the complex heavy ion optical potential which has been fully developed in Refs. 5-7 involves the following main steps:

(1) One starts from a realistic nucleon-nucleon interaction and solves the Bethe-Goldstone equation for the momentum configurations of two-sphere Fermi seas assumed to provide a local description of the heavy ion collisions.

(2) This yields a non-Hermitian $G$ matrix that one uses to construct ${ }^{5}$ a local effective nucleon-nucleon interaction $v^{c}(i, j)$ which is complex and depends on the collision energy as well as on the local matter and kinetic energy densities.

(3) The complex heavy ion optical potential for finite heavy ions at a separation distance $D$ between nuclear centers is then obtained ${ }^{6}$ by means of the standard ${ }^{8-13}$ formula

$$
V_{\text {opt }}(D)=\left\langle H^{c}(D)\right\rangle-\left\langle H^{c}(\infty)\right\rangle,
$$

which involves the ground state expectation values of the effective Hamiltonian

$$
H^{c}=\sum_{i} t_{i}+\frac{1}{2} \sum_{i \neq j} v^{c}(i, j)
$$

In the following the heavy ion optical potential corresponding to this program and calculated in Ref. 6 for ${ }^{16} \mathrm{O}+{ }^{16} \mathrm{O}$ will be used as a benchmark for testing various approximation schemes.

In Ref. 14 (hereafter referred to as I), we have exploited the similarity of $\left\langle H^{c}\right\rangle$ to a complex binding energy in order to extend the Negele-Vautherin density matrix expan$\operatorname{sion}^{15,16}$ (DME) to the complex domain. More precisely we have relied upon the approach developed in Ref. 15, where the DME was used both for the direct and exchange parts of the ground state expectation value. It was then found that the DME closely reproduced the imaginary part of the ${ }^{16} \mathrm{O}+{ }^{16} \mathrm{O}$ optical potential obtained in Ref. 6 at all separation distances. However, for the real part of $V_{\text {opt }}$, it turned out that the DME was unreliable in the inside region where nuclei overlap strongly, while for the physically important tail region it led to small but yet sizable differences with respect to the results of Ref. 6 .

Later work ${ }^{16,17}$ on static properties of nuclei has shown that the DME should only be used in the calculation of exchange contributions. The aim of this work was to investigate the validity of these considerations in the context of the heavy ion optical potential, and in particular to find out whether the above-mentioned discrepancies are to be ascribed to the failure of the DME for the direct part of $V_{\text {opt }}$ only. Moreover, the separation of $V_{\text {opt }}$ into its direct and exchange contributions also provides a new testing case (see Refs. 18-20 for other applications) for the Campi-Bouyssy prescription ${ }^{21,22}$ to accelerate the convergence of the exchange DME.

In Sec. II, we briefly recall the main features of the complex DME developed in I with due regard to the direct and exchange contributions to $V_{\text {opt }}$. We also point out the simplifications brought in by the Campi-Bouyssy prescription. Our results are presented and discussed in Sec. III.

\section{THE DIRECT AND EXCHANGE COMPLEX DME}

The DME approach to the complex heavy ion optical potential is based on the following truncated FourierBessel expansion for the density matrix:

$$
\begin{aligned}
\rho\left(\overrightarrow{\mathbf{R}}+\frac{\overrightarrow{\mathrm{s}}}{2}, \overrightarrow{\mathbf{R}}-\frac{\overrightarrow{\mathrm{s}}}{2}\right)= & \rho_{S L}(k s) \rho(\overrightarrow{\mathbf{R}})+\frac{35}{2 s k^{3}} j_{3}(k s) \\
& \times\left[\frac{1}{4} \nabla^{2} \rho(\overrightarrow{\mathbf{R}})-\tau^{(2)}(\overrightarrow{\mathbf{R}})+\frac{3}{5} k^{2} \rho(\overrightarrow{\mathbf{R}})\right],
\end{aligned}
$$

where $\rho(\vec{R})$ is the matter density at point $\vec{R}$ and $\rho_{S L}(k s)$ is defined by

$$
\rho_{S L}(k s)=\frac{3}{s k} j_{1}(k s) .
$$

Equation (2.1), moreover, involves $\tau^{(2)}(\overrightarrow{\mathbf{R}})$, which is the in- 
trinsic kinetic energy density at point $\vec{R}$ and momentum $k$. We refer to Eqs. (3.15) and (5.3)-(5.6) in I for the explicit definition of $\tau^{(2)}$. Here we just want to point out that $\tau^{(2)}$ has to replace the kinetic energy density $\tau$ which appears in the original expansion of Negele and Vautherin in order to preserve Galilean invariance. ${ }^{23}$ The momentum $k$ is in principle a free parameter. In $\mathrm{I}$, it was chosen as $^{15}$

$$
k=k_{F}=\left[\frac{3 \pi^{2}}{2} \rho(\overrightarrow{\mathbf{R}})\right]^{1 / 3} .
$$

Another choice proposed in Refs. 21 and 22 will be considered below.

When using uncritically an expansion similar to (2.1) for the product

$$
\rho\left[\overrightarrow{\mathbf{R}}+\frac{\overrightarrow{\mathrm{s}}}{2}\right] \rho\left[\overrightarrow{\mathbf{R}}-\frac{\overrightarrow{\mathrm{s}}}{2}\right)
$$

involved in the direct contribution to $\langle H\rangle$, one can explicitly write the potential part $\mathscr{H}_{\text {pot }}(\overrightarrow{\mathbf{R}})$ of the energy density as

$$
\mathscr{H}_{\text {pot }}(\overrightarrow{\mathbf{R}})=\mathscr{H}_{\text {pot }}^{D}(\overrightarrow{\mathbf{R}})+\mathscr{H}_{\text {pot }}^{E}(\overrightarrow{\mathbf{R}}),
$$

the direct and exchange part of $\mathscr{H}_{\text {pot }}$ being given by

$$
\begin{aligned}
& \mathscr{H}_{\mathrm{pot}}^{D}=A^{D}+B^{D} \tau^{(2)}+C^{D}(\vec{\nabla} \rho)^{2}+D^{D} \nabla^{2} \rho, \\
& \mathscr{H}_{\mathrm{pot}}^{E}=A^{E}+B^{E} \tau^{(2)}+C^{E}(\vec{\nabla} \rho)^{2}+D^{E} \nabla^{2} \rho,
\end{aligned}
$$

with

$$
\begin{aligned}
& A^{D}=V_{N M}^{D}, \\
& A^{E}=V_{N M}^{E}+\frac{3}{5}\left(\frac{3 \pi^{2}}{2}\right)^{2 / 3} \rho^{8 / 3} V^{E} \\
& B^{D}=0 \\
& B^{E}=-\rho V^{E} \\
& C^{D}=-\frac{1}{2} V^{D} \\
& C^{E}=0 \\
& D^{D}=\frac{1}{2} \rho V^{D}, \\
& D^{E}=\frac{1}{4} \rho V^{E}=-\frac{1}{4} B^{E}
\end{aligned}
$$

where by particularizing to a system with equal numbers of neutrons and protons, one has the following expressions for the spin and isospin dependent complex effective force defined in Ref. 5:

$$
\begin{gathered}
V_{N M}^{D}=\frac{\rho^{2}}{32} \int d^{3} s\left[3 v^{T E}(\dot{s})+3 v^{S E}(s)+9 v^{T 0}(s)+v^{S 0}(s)\right], \\
V_{N M}^{E}=\frac{\rho^{2}}{32} \int d^{3} s \rho_{S L}^{2}\left(k_{F} s\right)\left[3 v^{T E}(s)+3 v^{S E}(s)\right. \\
\left.-9 v^{T 0}(s)-v^{S 0}(s)\right]
\end{gathered}
$$

$V^{D}=\frac{35}{64} \int d^{3} s \frac{j_{3}\left(k_{F} s\right)}{k_{F}^{3} s}\left[3 v^{T E}(s)+3 v^{S E}(s)\right.$

$$
\left.+9 v^{T 0}(s)+v^{S 0}(s)\right] \text {, }
$$

$$
\begin{aligned}
V^{E}=\frac{35}{32} \int d^{3} s \rho_{S L}\left(k_{F} s\right) \frac{j_{3}\left(k_{F} s\right)}{k_{F}^{3} s}[ & 3 v^{T E}(s)+3 v^{S E}(s) \\
& \left.-9 v^{T 0}(s)-v^{S 0}(s)\right] .
\end{aligned}
$$

The coefficients $A, B, C$, and $D$ are complex and depend upon the relative momentum per nucleon $K_{r}$ and the local values of $\rho$ and $\tau^{(2)}$. As discussed in $I$, the dependence on $K_{r}$ and $\tau^{(2)}$ of the real parts of these coefficients is negligible, but the full dependence of the effective force $v^{S T}$ appears for the imaginary part of $A, B, C$, and $D$.

Relying on the results of Refs. 16 and 17, Campi and Bouyssy concentrated on the study of the exchange part of the energy density $\mathscr{H}$ only. They pointed out that one might hope to accelerate the convergence of the DME by choosing the free parameter $k$ in order to make the second term in Eq. (2.1) vanish. This can be done by taking

$$
k=k_{\mathrm{CB}}=\left\{\frac{5}{3 \rho(\overrightarrow{\mathbf{R}})}\left[\tau^{(2)}(\overrightarrow{\mathbf{R}})-\frac{1}{4} \nabla^{2} \rho(\overrightarrow{\mathbf{R}})\right]\right\}^{1 / 2} .
$$

Equation (2.7) then simplifies to

$$
\mathscr{H}_{\mathrm{pot}}^{E}(\mathrm{CB})=A^{E}(\mathrm{CB}) \text {, }
$$

with

$$
\begin{aligned}
& A^{E}(\mathrm{CB})= V_{N M}^{E}(\mathrm{CB}) \\
&=\frac{\rho^{2}}{32} \int d^{3} s \rho_{S L}^{2}\left(k_{\mathrm{CB}} s\right)\left[3 v^{T E}(s)+3 v^{S E}(s)\right. \\
&\left.\quad-9 v^{T 0}(s)-v^{S 0}(s)\right] .
\end{aligned}
$$

\section{RESULTS AND DISCUSSION}

Below we give results for the optical potential based on the prescriptions (2.3) and (2.20) for the local momentum $k$.

\section{A. $k=k_{F}$ [Equation (2.3)]}

The coefficients $A^{D}, \ldots, D^{E}$ which appear in Eqs. (2.6) and (2.7) have been computed for a series of two-sphere Fermi seas along the lines described in I, and the corresponding results have been fitted by using the following formulas ( $\mathscr{C}$ denotes any of the coefficients $\left.A^{D}, \ldots, D^{E}\right)$ :

$$
\begin{aligned}
& \operatorname{Im} \mathscr{C}=x\left(I_{1}+I_{2} x+I_{3} x^{2}\right), \\
& I_{n}=\rho^{\alpha}\left(I_{n 1}+I_{n 2} \rho+I_{n 3} \rho^{2}+I_{n 4} \rho^{3}\right) ; n=1,2,3, \\
& \operatorname{Re} \mathscr{C}=\rho^{\alpha}\left(R_{1}+R_{2} \rho+R_{3} \rho^{2}+R_{4} \rho^{3}\right),
\end{aligned}
$$

with

$$
x=\frac{\tau^{(2)}-\tau_{\min }^{(2)}}{\tau_{\max }^{(2)}-\tau_{\min }^{(2)}}
$$




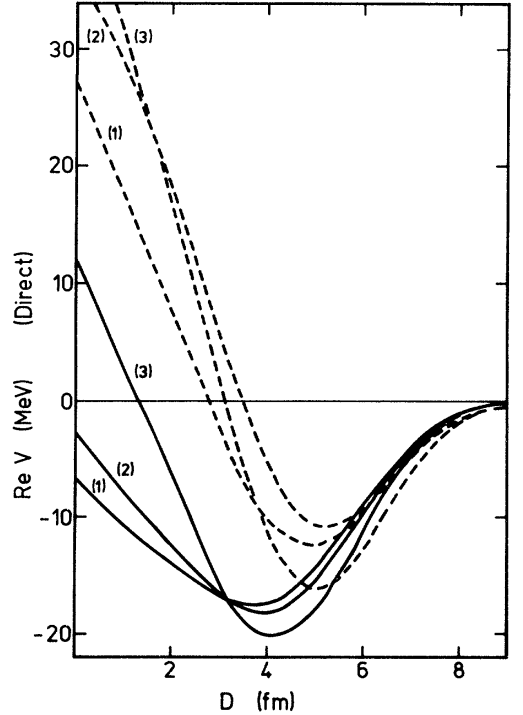

FIG. 1. Direct contribution to the real part of the ${ }^{16} \mathrm{O}+{ }^{16} \mathrm{O}$ optical potential. The full curves correspond to the DME with $k=k_{F}$ [Eq. (2.3)]. The dashed curves correspond to the results of Ref. 6. The notations (1), (2), and (3) correspond to $K_{r}=0$, 0.5 , and $1 \mathrm{fm}^{-1} /$ nucleon, respectively. The contribution due to the kinetic energy part of $H^{c}$ [Eq. (1.2)] is not included.

and

$$
\alpha=2,1,0 \text {, and } 1
$$

for $A, B, C$, and $D$, respectively.

In Eq. (3.4), $\tau_{\min }^{(2)}$ and $\tau_{\max }^{(2)}$ are the minimum and maximum values allowed for $\tau^{(2)}$ when the matter density $\rho$ is fixed. At each integration point in Eq. (1.1), the local

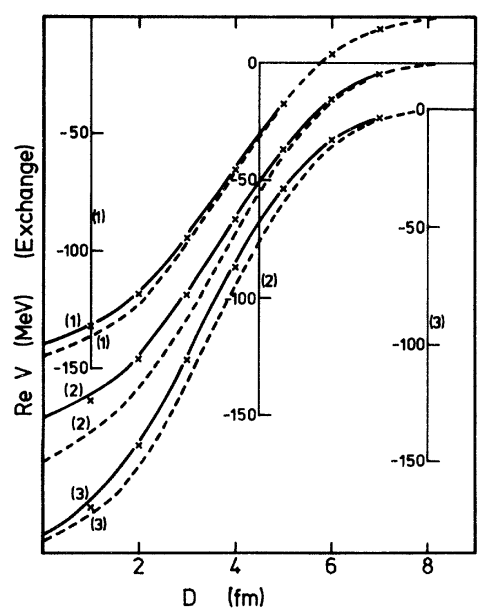

FIG. 2. Exchange contribution to the real part of the ${ }^{16} \mathrm{O}+{ }^{16} \mathrm{O}$ optical potential. The full curves are the DME results with $k=k_{F}$ [Eq. (2.3)], and the dashed ones those of Ref. 6. The crosses represent the DME calculations with $k=k_{\mathrm{CB}}$ [Eq. (2.20)]. The notations (1), (2), and (3) correspond to $K_{r}=0,0.5$, and $1 \mathrm{fm}^{-1} /$ nucleon, respectively. The ordinate scales have been shifted for clarity.

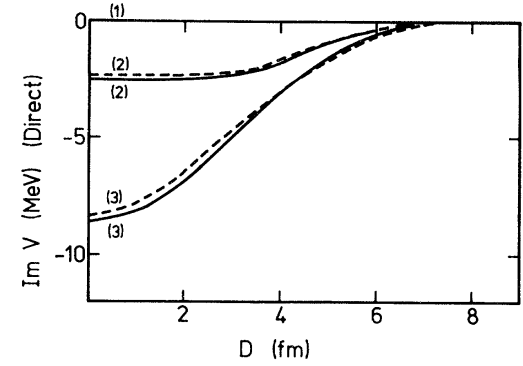

FIG. 3. Same as Fig. 1 but for the direct contribution to the imaginary part of the ${ }^{16} \mathrm{O}+{ }^{16} \mathrm{O}$ optical potential.

values of $\rho$ and $\tau^{(2)}$ are computed from the two center harmonic oscillator shell models described in I.

The direct and exchange contributions to the real potential part of $V_{\text {opt }}$ correspond to the full curves in Figs. 1 and 2, respectively. The dashed curves correspond to the results of Ref. 6 . Notice that the above-mentioned discrepancies in the tail region of the total (i.e., direct plus exchange) $\operatorname{Re} V_{\text {opt }}$ are to be entirely ascribed to the direct part of $\operatorname{Re} V_{\text {opt }}$. Indeed, for the exchange part of $\operatorname{Re} V_{\text {opt }}$, the DME yields results which coincide with the exchange part of the exact $\operatorname{Re} V_{\text {opt }}$ in the tail and remain very good down to zero separation distance. For the imaginary part of $V_{\text {opt }}$, the DME yields good results both for the direct (Fig. 3) and the exchange (Fig. 4) contributions. As emphasized in $I$, the kinetic energy density effects rule the imaginary part of the optical potential, and the alteration of the saturation properties of nuclear matter which are responsible for the discrepancies in the real part of $V_{\text {opt }}$ play no role for $\operatorname{Im} V_{\text {opt }}$. Let us also remark that the DME introduces errors of opposite signs for the direct and exchange parts of both $\operatorname{Re} V_{\text {opt }}$ and $\operatorname{Im} V_{\text {opt }}$.

For future reference, we give in Table I the exchange $I_{n k}$ and $R_{n}$ fit coefficients for three values of the relative momentum per nucleon $K_{r}$.

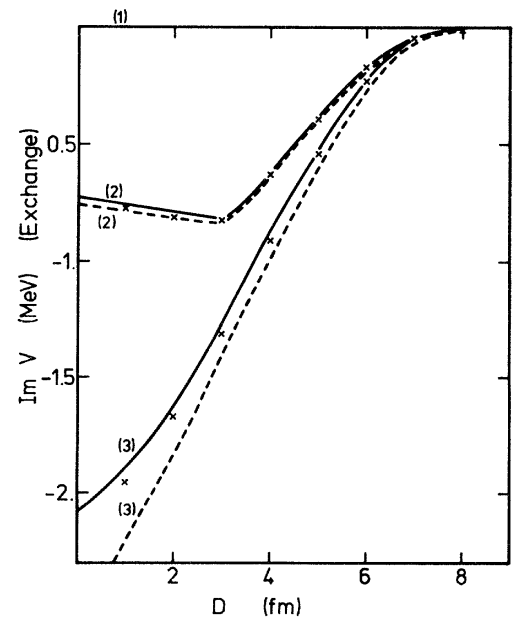

FIG. 4. Same as Fig. 2 but for the exchange contribution to the imaginary part of the ${ }^{16} \mathrm{O}+{ }^{16} \mathrm{O}$ optical potential. 
TABLE I. The real $R_{j}$ and imaginary $I_{n j}$ fit parameters [Eqs. (3.1)-(3.3)] for the exchange DME coefficients $A^{E}$ and $B^{E}$ [Eqs. (2.9), (2.11), and (2.15)]. Note that $C^{E}$ [Eq. (2.13)] vanishes identically, that $D^{E}=-\frac{1}{4} B^{E}$ [Eq. (2.15)], and that $R_{j}$ does not depend upon $K_{r}$.

\begin{tabular}{|c|c|c|c|c|c|c|}
\hline $\mathscr{C}$ & & & $j=1$ & $j=2$ & $j=3$ & $j=4$ \\
\hline \multirow[t]{7}{*}{$A^{E}$} & & $R_{j}$ & -342.91 & 806.42 & -2470.8 & 3162.4 \\
\hline & $K_{r}=0.5$ & $I_{1 j}$ & -1.0333 & -4.0460 & 51.665 & -93.184 \\
\hline & & $I_{2 j}$ & 0.15702 & 12.456 & -80.714 & 127.67 \\
\hline & & $I_{3 j}$ & 0.40130 & -13.947 & 75.155 & -111.82 \\
\hline & $K_{r}=1.0$ & $I_{1 j}$ & -13.282 & 123.86 & -409.02 & 462.74 \\
\hline & & $I_{2 j}$ & 20.461 & -227.52 & 858.97 & -1074.5 \\
\hline & & $I_{3 j}$ & -16.832 & 206.67 & -833.28 & 1092.0 \\
\hline \multirow[t]{7}{*}{$B^{E}$} & & $R_{j}$ & 178.24 & -1058.2 & 3359.3 & -3969.4 \\
\hline & $K_{r}=0.5$ & $I_{1 j}$ & 0.89300 & -1.6991 & -13.110 & 32.117 \\
\hline & & $I_{2 j}$ & -0.30740 & -4.2111 & 34.202 & -57.614 \\
\hline & & $I_{3 j}$ & -0.12053 & 6.5022 & -36.914 & 56.152 \\
\hline & $K_{r}=1.0$ & $I_{1 j}$ & 9.1548 & -91.622 & 319.86 & -376.59 \\
\hline & & $I_{2 j}$ & -14.065 & 163.45 & -634.96 & 808.30 \\
\hline & & $I_{3 j}$ & 11.292 & -141.52 & 578.26 & -763.18 \\
\hline
\end{tabular}

\section{B. $k=k_{\mathrm{CB}}$ [Equation (2.20)]}

Since we will now concentrate on the exchange part of $V_{\text {opt }}$, we only need calculate the coefficient $A^{E}(\mathrm{CB})$ of Eq. (2.21) for a series of Fermi seas and a series of $k_{\mathrm{CB}}$ values. The dependence of $A^{E}(\mathrm{CB})$ on $\rho$ and $\tau^{(2)}$ has also been fitted according to formulas (3.1)-(3.3). We did not use a polynomial fit for the $k_{\mathrm{CB}}$ dependence of $A^{E}(\mathrm{CB})$, which we display in Fig. 5 for $K_{r}=0.5 \mathrm{fm}^{-1}$ nucleon, $\rho=0.17$ nucleon $/ \mathrm{fm}^{3}$, and $\tau^{(2)}=0.19109 \mathrm{fm}^{-5}\left(\hbar^{2} / 2 m=1\right)$. Instead we have interpolated between the values of $A^{E}(\mathrm{CB})$ computed at the mesh points $k_{\mathrm{CB}}=0(0.1) 2$ by means of a four-point Lagrange formula. The above $k_{\mathrm{CB}}$ range is large enough to include the local values of $k_{\mathrm{CB}}$ which arise in the calculation of the ${ }^{16} \mathrm{O}+{ }^{16} \mathrm{O}$ optical potential. This can be seen, for instance, from Fig. 6, where we display the local values of $k_{\mathrm{CB}}$ along the axis joining the centers of the two colliding ${ }^{16} \mathrm{O}$ ions. For symmetry

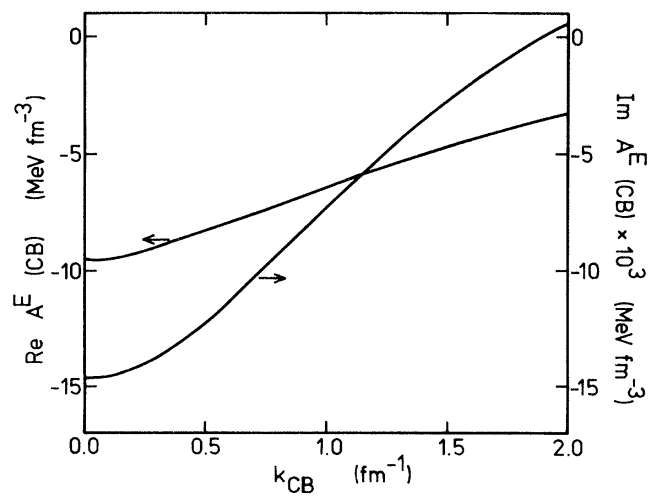

FIG. 5. Real and imaginary parts of the $A^{E}(\mathrm{CB})$ coefficient [Eq. (2.21)] as a function of $k_{\mathrm{CB}}$ for $K_{r}=0.5 \mathrm{fm}^{-1} /$ nucleon, $\rho=0.17$ nucleon $/ \mathrm{fm}^{3}$, and for an intermediate value of $\tau^{(2)}=0.19109 \mathrm{fm}^{-5}\left(\hbar^{2} / 2 m=1\right)$. For the above-mentioned $K_{r}$ and $\rho$, one has $\tau_{\min }^{(2)}=0.18858$ and $\tau_{\max }^{(2)}=0.19179$. reasons we have represented the positive part of the $z$ axis only.

Notice that contrary to $k_{F}$ [Eq. (2.3)], $k_{\mathrm{CB}}$ does not vanish with the density. This is due ${ }^{21}$ to our use of harmonic oscillator single particle wave functions. It also appears that the dependence of $k_{\mathrm{CB}}$ on $K_{r}$ varies with the separation distance $D$ between nuclei, leading to a more pronounced dependence for stronger overlaps, as expected from the Pauli principle.

Our results for the exchange part of $V_{\text {opt }}$ correspond to the crosses in Figs. 2 and 4. It turns out that the CampiBouyssy prescription yields corrections which do go in the right direction, but they remain too small to completely cancel the discrepancies remaining between the exchange part of $V_{\text {opt }}$ computed with the DME and that computed in Ref. 6 with a finite range interaction.

As mentioned above, the Campi-Bouyssy approxima-

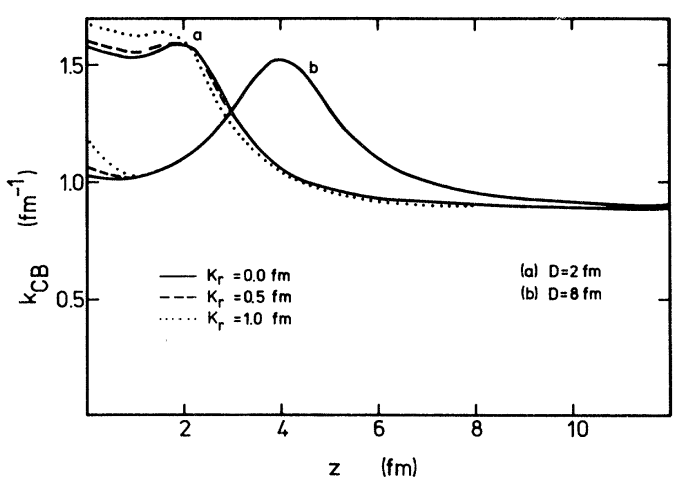

FIG. 6. The Campi-Bouyssy local momentum $k_{\mathrm{CB}}$ along the positive $z$ axis joining the centers of the colliding ${ }^{16} \mathrm{O}$ ions. The origin $z=0$ is located at mid-distance of the centers of the ${ }^{16} \mathrm{O}$ ions. The labels $a$ and $b$ correspond to the separation distances $D=2$ and $8 \mathrm{fm}$, respectively. The full, dashed, and dotted curves are results for $K_{r}=0,0.5$, and $1 \mathrm{fm}^{-1} /$ nucleon, respectively. 
tion was devised to accelerate the convergence of the exchange part of the DME whatever treatment-exact or approximate-is applied to the direct part. This means that if DME is used both for the direct and the exchange terms, it is not necessary to take the same value of $k$ in both terms. Nevertheless, for the sake of completeness, we have also calculated the direct term with $k=k_{\mathrm{CB}}$ in the DME approximation. We found that the direct part of $\operatorname{Im} V_{\text {opt }}$ remains practically insensitive to the choice of $k$. As far as the direct real part is concerned, it turned out that the Campi-Bouyssy local momentum increases the disagreement between the DME results and those of Ref. 6 by various amounts, depending on $D$ and $K_{r}$. In the tail region, however, the results remain close to those obtained with $k=k_{F}$.
To summarize, the present work supports the validity of the DME limited to the exchange part of the optical potential. In the physically relevant tail region, the exchange DME can be considered as an extremely good approximation. As far as the direct contribution is concerned, the DME remains safe for the imaginary part of $V_{\text {opt }}$, while it is unreliable, even in the tail region, for $\operatorname{Re} V_{\text {opt }}$. Hence in the present context, there seems to be no need for improving the exchange part of $V_{\text {opt }}$ in the tail region. On the other hand, at shorter separation distances, the Campi-Bouyssy prescription slightly improves the DME calculations.

We thank Dr. X. Campi for useful correspondence.
${ }^{1}$ D. A. Saloner and C. Toepffer, Nucl. Phys. $\underline{\text { A283, }} 108$ (1977).

${ }^{2}$ F. Beck, K. H. Müller, and H. S. Köhler, Phys. Rev. Lett. $\underline{40}$, 837 (1978).

${ }^{3}$ T. Izumoto, S. Krewald, and A. Faessler, Nucl. Phys. A341, 319 (1980).

${ }^{4}$ T. Izumoto, S. Krewald, and A. Faessler, Phys. Lett. 95B, 16 (1980).

${ }^{5}$ A. Faessler, T. Izumoto, S. Krewald, and R. Sartor, Nucl. Phys. A359, 509 (1981).

${ }^{6}$ R. Sartor, A. Faessler, and S. B. Khadkikar, Nucl. Phys. A359, 467 (1981).

${ }^{7}$ S. B. Khadkikar, L. Rikus, A. Faessler, and R. Sartor, Nucl. Phys. A369, 495 (1981).

${ }^{8}$ K. A. Brueckner et al., Phys. Rev. 171, 1188 (1968).

${ }^{9}$ K. A. Brueckner et al., Phys. Rev. 173, 944 (1968).

${ }^{10}$ D. M. Brink and Fl. Stancu, Nucl. Phys. A243, 175 (1975).

${ }^{11}$ Fl. Stancu and D. M. Brink, Nucl. Phys. $\underline{\mathrm{A270}}, 236$ (1976).
${ }^{12}$ C. Ngô et al., Nucl. Phys. A240, 353 (1975).

${ }^{13}$ C. Ngô et al., Nucl. Phys. A252, 237 (1975).

${ }^{14}$ R. Sartor and Fl. Stancu, Phys. Rev. C 26, 1025 (1982).

15J. W. Negele and D. Vautherin, Phys. Rev. C $\underline{5}, 1472$ (1972).

16J. W. Negele and D. Vautherin, Phys. Rev. C 11, 1031 (1975).

${ }^{17}$ D. W. L. Sprung, M. Vallières, X. Campi, and Che-Ming Ko, Nucl. Phys. A253, 1 (1975).

${ }^{18}$ N. Vinh Mau, Phys. Lett. 71B, 5 (1977).

${ }^{19}$ R. K. Bhaduri and D. W. L. Sprung, Nucl. Phys. $\underline{\text { A297, } 365}$ (1978).

${ }^{20}$ F. Duggan, M. Lassaut, F. Michel, and N. Vinh Mau, Nucl. Phys. A355, 141 (1980).

${ }^{21}$ X. Campi and A. Bouyssy, Phys. Lett. 73B, 263 (1978).

${ }^{22}$ A. Bouyssy and X. Campi, Nukleonika 24, 1 (1979).

${ }^{23}$ Y. M. Engel, D. M. Brink, K. Goeke, S. J. Krieger, and D. Vautherin, Nucl. Phys. A249, 215 (1975). 